Pacific Journal of Mathematic 


\title{
ON CERTAIN SUMS GENERATING THE DEDEKIND SUMS AND THEIR RECIPROCITY LAWS
}

\author{
M. MiKOLÁS
}

1. Introduction. Let $\{u\}=u-[u]$ denote the fractional part of $u$ and let $((u))=\{u\}-\frac{1}{2}$. Dedekind sums are defined for example, by

$$
s_{1}(h, k)=\sum_{\lambda=0}^{k-1}\left(\left(\frac{\lambda}{k}\right)\right)\left(\left(\frac{\lambda h}{k}\right)\right)
$$

where $h$ and $k$ are relatively prime positive integers. These sums which were studied by Dedekind [7], and more recently by Rademacher and Whiteman [9], [12] in connection with the theory of the modular function $\eta(\tau)$, occur also in the theory of partitions and in a great number of special papers. (Cf. for example [1]-[13].) The most important property of $s_{1}(h, k)$ is the reciprocity law

$$
s_{1}(h, k)+s_{1}(k, h)=\left(h^{2}+3 h k+k^{2}+1\right) /(12 h k) .
$$

A few years ago, Apostol [1] (for $r=\nu$ ) and Carlitz [3] introduced and investigated the so-called generalized Dedekind sums

$$
s_{r}^{(\nu)}(h, k)=\sum_{\lambda=0}^{k-1} P_{\nu+1-r}\left(\begin{array}{c}
\lambda \\
k
\end{array}\right) P_{r}\left(\frac{\lambda h}{k}\right) \quad 0 \leqq r \leqq \nu+1,
$$

$P_{r}$ denoting the well-known Bernoulli function defined by the expansion

$$
z e^{u z} /\left(e^{z}-1\right)=\sum_{n=0}^{\infty} P_{n}(u) z^{n} / n ! \quad|z|<2 \pi
$$

for $0 \leqq u<1$ and by $P_{r}(u)=P_{r}(\{u\})$ for $u$ arbitrary real. They found the corresponding extensions of (1.2) too.

Now, we shall continue to develop these results in two directions. Next we give a systematic treatment of certain exponential sums (2.1), (2.3) generating

$$
\mathfrak{S}_{m, n}\left(\begin{array}{cc}
a & b \\
c
\end{array}\right)=\sum_{\nu=0}^{c-1} P_{m}\left(\frac{\lambda a}{c}\right) P_{n}\left(\frac{\lambda b}{c}\right) \quad m, n=0,1,2, \cdots
$$

with $(a, c)=(b, c)=1, c>0$. We obtain (among others) a three-term relation of new type (Theorem 1) which implies (in extended form) all the above reciprocity theorems (see (5.1)-(5.10)). Let us remark that the sum function (2.5) with other notations is also used in [6]. On the other hand, we get a functional equation for

Received July 31, 1956. 


$$
\mathfrak{D}_{c}^{a, b}(w, z)=\sum_{\lambda=1}^{c-1} \zeta\left(w,\left\{\frac{\lambda a}{c}\right\}\right) \zeta\left(z,\left\{\frac{\lambda b}{c}\right\}\right)
$$

where $\zeta(s, u)$ is the Hurwitz zeta function (Theorem 2). By

$$
\zeta(1-n, u)=-P_{n}(u) / n \quad 0<u \leqq 1 ; n=1,2, \cdots,
$$

(1.5) can be regarded substantially as a (transcendental) generalization of (1.4).

2. Preliminaries on $\mathfrak{S}_{c}^{a, b}(x, y), \mathfrak{\Im}_{m, n}\left(\begin{array}{c}a b \\ c\end{array}\right)$. In what follows, $x, y$, $w, z$ denote complex variables, $a, b$ and $c$ are integers and $c>0$; for brevity we write, as usual, $e(z)=e^{2 \pi i z}$.

Let us put

$$
S_{c}^{a, b}(x, y)=\sum_{\lambda(\bmod c)} e\left(\left\{\frac{\lambda a}{c}\right\} x+\left\{\frac{\lambda b}{c}\right\} y\right)
$$

with $(a, c)=(b, c)=1$, the summation extending over a complete residue system modulo $c$. It is obvious that (2.1) is independent of the choice of this residue system ${ }^{1}$ and for $a=b$ or $c=1,2$ it is independent of $a, b$. The function $S_{c}^{a, b}(x, y)$ remains unaltered if we change $a, b$ or $x, y$ by multiplies of $c$. By this periodicity, it is no restriction to suppose for example, that $0 \leqq \Re(x)<c,-c<\Re(y) \leqq 0$.

We have $S_{c}^{a, b}(x, y)=S_{c}^{b, a}(y, x)$ and

$$
S_{c}^{-a, b}(x, y)=e(x) S_{c}^{a, b}(-x, y)+1-e(x),
$$

since $\{-u\}=0$ or $1-\{u\}$ according as $u$ is an integer or not.

The function

$$
\Im_{c}^{a, b}(x, y)=[e(x)-1]^{-1}[e(y)-1]^{-1} S_{c}^{a, b}(x, y) \quad x, y \neq 0, \pm 1, \cdots
$$

has corresponding trivial properties ; in particular, (2.2) implies

$$
\Im_{c}^{-a, b}(x, y)=-\Im_{c}^{a, b}(-x, y)-[e(y)-1]^{-1} .
$$

By the definition of Bernoulli functions and (1.4) we obtain

$$
x y \mathfrak{S}_{c}^{a, b}(x / 2 \pi i, y / 2 \pi i)=\sum_{m, n=0}^{\infty} \frac{x^{m} y^{n}}{m ! n !} \mathfrak{\Xi}_{m, n}\left(\begin{array}{cc}
a & b \\
\epsilon
\end{array}\right) \quad|x|,|y|<2 \pi .
$$

\section{Here}

${ }^{1}$ Hence we see that $S_{c}^{a, b}(x, y)=S_{c}^{1, b^{\prime}}(x, y)$ for a suitable integer $b^{\prime}$; however, the above symmetric notation seems the most convenient. 


$$
\mathfrak{S}_{0, n}\left(\begin{array}{cc}
a & b \\
c
\end{array}\right)=\sum_{l=0}^{c-1} P_{n}\left(\begin{array}{l}
l \\
c
\end{array}\right)=c^{1-n} B_{n} \quad n=0,1, \cdots
$$

$B_{n}=P_{n}(0)$ denoting the Bernoullian numbers.

Note that $\mathfrak{S}_{m, n}\left(\begin{array}{cc}a & b \\ c\end{array}\right)=\mathfrak{I}_{n, m}\left(\begin{array}{cc}b & a \\ c\end{array}\right)$ and $\mathfrak{I}_{m, n}\left(\begin{array}{cc}a & a \\ c\end{array}\right)$ does not depend on $a$; especially we have $\mathfrak{S}_{m, n}\left(\begin{array}{cc}1 & b \\ c\end{array}\right)=\mathfrak{S}_{n}^{(m+n-1)}(b, c)$, furthermore

$$
\begin{array}{r}
\mathfrak{S}_{m, n}\left(\begin{array}{cc}
a & b \\
1
\end{array}\right)=B_{m} B_{n}, \quad \mathfrak{S}_{m, n}\left(\begin{array}{cc}
a & b \\
2
\end{array}\right)=B_{m} B_{n}\left[1+\left(1-2^{1-m}\right)\left(1-2^{1-n}\right)\right] \\
m, n=0,1, \cdots .
\end{array}
$$

3. Representation by cotangents and Eulerian numbers respectively. Let $c>1$. The identity

$$
\sum_{\mu=0}^{c-1} e\left(\frac{\mu x}{c}\right) e\left(\frac{\mu \nu}{c}\right)=[e(x)-1]\left[e\left(\frac{x+\nu}{c}\right)-1\right]^{-1}
$$

yields after multiplication by $e\left(-\frac{\mu \nu}{c}\right)(\nu=0,1, \cdots, c-1)$ and summation

$$
\begin{aligned}
e\left(\frac{\mu x}{c}\right)=\frac{1}{c}[e(x)-1] \sum_{\nu=0}^{c-1}\left[e\left(\frac{x+\nu}{c}\right)-1\right]^{-1} e\left(-\frac{\mu \nu}{c}\right) & \\
\mu & =0,1, \cdots, \nu-1 ;
\end{aligned}
$$

(3.1) and (3.2) hold clearly provided that $(x+\nu) / c$ is not an integer $(\nu=0,1, \cdots, c-1)$. Hence by putting $\mu=c\{a \lambda / c\}, a$ and $c$ being coprime we get

$$
e\left(x\left\{\frac{a \lambda}{c}\right\}\right)=\frac{1}{c}[e(x)-1] \sum_{\nu=0}^{c-1}\left[e\left(\frac{x+\nu}{c}\right)-1\right]^{-1} e\left(-\nu \frac{a \lambda}{c}\right) .
$$

Furthermore, by using the corresponding expression for $e(y\{b \lambda / c\})$, $(b, c)=1$,

$$
\begin{aligned}
& S_{c}^{a, b}(x, y)=\frac{1}{c^{2}}[e(x)-1][e(y)-1] \sum_{p, q(\bmod c)}\left[e\left(\frac{x+p}{c}\right)-1\right]^{-1}\left[e\left(\frac{y+q}{c}\right)-1\right]^{-1} \\
& \times \sum_{\lambda=0}^{c-1} e\left(-\frac{\lambda(a p+b q)}{c}\right) .
\end{aligned}
$$

If we consider the complete residue systems $(\bmod c): p=-b r, q=a \rho$ $(r, \rho=0,1, \cdots, c-1)$ and take into account that $\sum_{\lambda=0}^{c-1} e\left(-\lambda \frac{a b(\rho-r)}{c}\right)$ 
vanishes except for $\rho=r$ when it has the value $c$, it follows simply that

$$
\mathfrak{S}_{c}^{a, b}(x, y)=\frac{1}{c} \sum_{r(\bmod c)}\left[e\left(\begin{array}{c}
x-b r \\
c
\end{array}\right)-1\right]^{-1}\left[e\left(\begin{array}{c}
y+a r \\
c
\end{array}\right)-1\right]^{-1},
$$

holds for all $x, y \neq 0, \pm 1, \cdots$ and, because of the definition (2.3), in the case $c=1$ too. By $[1-e(z)]^{-1}=\frac{1}{2}(1+i$ ctg $\pi z)$ and

$$
\sum_{\mu=0}^{c-1} \operatorname{ctg} \pi\left(z+\frac{\mu}{c}\right)=c \cdot \operatorname{ctg} c \pi z,
$$

we have the equivalent formula:

$$
\begin{aligned}
\subseteq_{c}^{a, b}(x, y)=\frac{1}{4} \cdot[1 & +i(\operatorname{ctg} \pi x+\operatorname{ctg} \pi y)] \\
& -\frac{1}{4 c} \sum_{r(\bmod c)} \operatorname{ctg} \pi \frac{x-b r}{c} \operatorname{ctg} \pi \frac{y+a r}{c} ;
\end{aligned}
$$

(3.4) or (3.5) expresses the sum (2.3) by means of periodic elementary functions, without using the arithmetical function $\{u\}$.

(3.4) leads immediately to corresponding representations of $\mathfrak{B}_{m, n}\left(\begin{array}{cc}a & b \\ c\end{array}\right)$ by means of the so-called Eulerian numbers $H_{n}\left(\eta^{k}\right)$, defined for a root of unity $\eta^{k}=e\left(\frac{k}{c}\right), c>1, c \nmid k$ by

$$
\left(1-\eta^{k}\right) /\left(e^{z}-\eta^{k}\right)=\sum_{n=0}^{\infty} H_{n}\left(\eta^{k}\right) z^{n} / n ! \quad|z|<2 \pi\{k / c\} .
$$

In fact, after expanding the right-hand members of

$$
\begin{aligned}
x y \Im_{c}^{a, b}(x / 2 \pi i, y / 2 \pi i)= & (x y / c)\left(e^{x / c}-1\right)^{-1}\left(e^{y / c}-1\right)^{-1} \\
& +(x y / c) \sum_{r=1}^{c-1}\left(e^{x / c} \eta^{-b r}-1\right)^{-1}\left(e^{y / c} \eta^{a r}-1\right)^{-1},
\end{aligned}
$$

we find

$$
\begin{gathered}
x y \Im_{c}^{a, b}(x / 2 \pi i, y / 2 \pi i)=c+\sum_{n=1}^{\infty} \frac{B_{n}}{n ! c^{n-1}}\left(x^{n}+y^{n}\right) \\
+\sum_{m, n=1}^{\infty} \frac{x^{m} y^{n}}{m ! n ! c^{m+n-1}}\left[B_{m} B_{n}+m n \sum_{r=1}^{c-1} \frac{H_{m-1}\left(\eta^{b r}\right) H_{n-1}\left(\eta^{-a r}\right)}{\left(\gamma^{a r}-1\right)\left(\eta^{-b r}-1\right)}\right] \quad|x|,|y|<{ }_{c}^{2 \pi},
\end{gathered}
$$

so that comparison with (2.5) gives in addition to (2.6)

$$
\begin{array}{r}
\mathfrak{S}_{m, n}\left(\begin{array}{cc}
a & b \\
c
\end{array}\right)=\frac{1}{c^{m+n-1}}\left[B_{m} B_{n}+m n \sum_{r=1}^{c-1} \frac{H_{m-1}\left(\eta^{b r}\right) H_{n-1}\left(\eta^{-a r}\right)}{\left(r^{a r}-1\right)\left(\eta^{-b r}-1\right)}\right] \\
m, n=1,2, \cdots,
\end{array}
$$


a formula implying a result of Carlitz [3, (6.5)]. In particular, for $m=n=1$ (3.8) becomes

$$
\begin{aligned}
\mathfrak{Q}_{11}\left(\begin{array}{cc}
a & b \\
c
\end{array}\right) & =\frac{1}{4 c}+\frac{1}{c} \sum_{r=1}^{c-1}\left(\eta^{a r}-1\right)^{-1}\left(\eta^{-b r}-1\right)^{-1} \\
& =\frac{1}{4}+\frac{1}{4 c} \sum_{r=1}^{c-1} \operatorname{ctg} \frac{\pi a r}{c} \operatorname{ctg} \frac{\pi b r}{c},
\end{aligned}
$$

which contains two equivalent representations due to Rademacher and Rédei (for $a=1$; cf. for example, [4], (2.2) and [2], (5) respectively).

4. The main property of $\mathfrak{S}_{c}^{a, b}(x, y)$. Our next purpose is to deduce a peculiar symmetry relation relating to the sums in question, by applying the calculus of residues.

THEOREM 1. We have for $a, b, c$ positive, mutually coprime, and for $0 \leqq \Re(x)<1,-1<\Re(y) \leqq 0$ the relation

$$
\begin{aligned}
\mathfrak{S}_{b}^{c, a}(a x+b y,-c x)+\Im_{c}^{a, b}(c x, c y) & +\mathfrak{S}_{a}^{b, c}(-c y, a x+b y) \\
& =[1-e(a x+b y)]^{-1},
\end{aligned}
$$

provided that $a x+b y$, cx and cy are not integers.

Proof. We consider the integral

$$
\mathfrak{F}=\frac{1}{2 \pi i} \int_{Q}[e(z)-1]^{-1}\left[e\left(x-\frac{b}{c} z\right)-1\right]^{-1}\left[e\left(y+\frac{a}{c} z\right)-1\right]^{-1} d z
$$

the path of integration being a rectangle whose vertices are the points $-\varepsilon \pm t i, c-\varepsilon \pm t i$ with

$$
t>\max \left\{\frac{c}{b}|\Im(x)|, \frac{c}{a}|\Im(y)|\right\}
$$

and

$$
0<\varepsilon<\min \left\{\begin{array}{l}
c \\
b
\end{array}(1-\Re(x)), \frac{c}{a}(1+\Re(y))\right\},
$$

taken in positive direction. A straight-forward calculation shows that only singularities of the integrand inside $Q$ are at the points:

$$
\begin{array}{ll}
z=\lambda & \lambda=0,1, \cdots, c-1 ; \\
z=\frac{c}{b}(\mu+x) & \mu=0,1, \cdots, b-1 ; \\
z={ }_{a}^{c}(\nu-y) & \nu=0,1, \cdots, a-1 ;
\end{array}
$$


by our assumptions, these are all distinct and poles of order 1 only of the first, second, and third factor respectively. Since

$$
\begin{aligned}
& \quad \underset{z=\lambda}{\operatorname{res}}[e(z)-1]^{-1}=1 / 2 \pi i \\
& \operatorname{res}_{z=(c / b)(\mu+x)}[e(x-b z / c)-1]^{-1}=-c / 2 \pi i b, \\
& \underset{z=(c / a)(\nu-y)}{\operatorname{res}}[e(y+a z / c)-1]^{-1}=c / 2 \pi i a,
\end{aligned}
$$

the residue theorem yields

$$
\begin{aligned}
& 2 \pi i \cdot \widetilde{\gamma}=\sum_{\lambda=0}^{c-1}\left[e\left(x-\frac{\lambda b}{c}\right)-1\right]^{-1}\left[e\left(y+\begin{array}{c}
\lambda a \\
c
\end{array}\right)-1\right]^{-1} \\
& -\frac{c}{b} \sum_{\mu=0}^{b-1}\left[e\left(\frac{a}{b} x+y+\frac{\mu a}{b}\right)-1\right]^{-1}\left[e\left(\begin{array}{l}
c \\
b
\end{array} x+\frac{\mu c}{b}\right)-1\right]^{-1} \\
& +\frac{c}{a} \sum_{\nu=0}^{a-1}\left[e\left(x+\frac{b}{a} y+\frac{\nu b}{a}\right)-1\right]^{-1}\left[e\left(-\frac{c}{a} y+\frac{\nu c}{a}\right)-1\right]^{-1}
\end{aligned}
$$

and therefore, by (3.4), we obtain

$$
\mathfrak{S}_{c}^{a, b}(c x, c y)-\Im_{b}^{c,-a}(a x+b y, c x)+\Im_{a}^{c, b}(a x+b y,-c y)=(2 \pi i / c) \mathfrak{r} .
$$

Now, if we write

$$
\int_{Q}=\int_{c-\varepsilon-t i}^{c-\varepsilon+t i}+\int_{c-\varepsilon+t i}^{-\varepsilon+t i}+\int_{-\varepsilon+t i}^{-\varepsilon-t i}+\int_{-\varepsilon-t i}^{c-\varepsilon-t i}
$$

with the integrand of (4.2) and straight-line paths, the sum of the first and third member on the right vanishes because of the periodicity (with period $c$ ) of

$$
[e(z)-1]^{-1}[e(x-b z / c)-1]^{-1}[e(y+a z / c)-1]^{-1} .
$$

On the other hand, using the estimate $|e(u+i v)-1| \geqq\left|e^{-2 \pi v}-1\right|(u, v$ arbitrary real), we find at once that the integrals along the horizontal segments tend to zero as $t \rightarrow \infty$. Hence (4.3) implies for $t \rightarrow \infty$

$$
\mathfrak{S}_{a}^{c, b}(a x+b y,-c y)-\Im_{b}^{c,-a}(a x+b y, c x)+\Im_{c}^{a, b}(c x, c y)=0
$$

which is, by (2.4), equivalent to (4.1).

5. Applications; extension of the well-known reciprocity theorems. (1) If we write

$$
\mathfrak{T}_{c}^{a, b}(x, y)=\frac{1}{c} \sum_{r(\bmod c)} \operatorname{ctg} \pi^{x-b r} \frac{\operatorname{ctg} \pi^{y+a r}}{c}
$$

and use (3.5), then (4.1) becomes 


$$
\mathfrak{I}_{b}^{c, a}(a x+b y,-c x)+\widetilde{I}_{c}^{a, b}(c x, c y)+\mathfrak{I}_{a}^{b, c}(-c y, a x+b y)=1 .
$$

By (3.9), this may be regarded as a generalization of the reciprocity theorem of Dedekind sums. For, by putting $y=-x$ in (5.2) and making $x \rightarrow 0$, we obtain on the basis of the Laurent expansion $\operatorname{ctg} z=z^{-1}-\frac{1}{3} z-\cdots$

$$
\mathfrak{P}_{11}\left(\begin{array}{cc}
b & c \\
a
\end{array}\right)+\mathfrak{S}_{11}\left(\begin{array}{cc}
c & a \\
b
\end{array}\right)+\mathfrak{S}_{11}\left(\begin{array}{cc}
a & b \\
c
\end{array}\right)=\frac{1}{2}+\frac{1}{12}\left(\begin{array}{c}
a \\
b c
\end{array}+\frac{b}{c a}+\frac{c}{a b}\right)
$$

a remarkably symmetric three-term relation which for $a=1$ reduces to (1.2) with $h=b, k=c$. (Cf. also a result of Rademacher in [11].)

(2) Let us replace in (4.1) $x, y$ by $x / 2 \pi i$ and $y_{i}^{\prime} 2 \pi i$ respectively, multiply both sides by $c^{2} x y(a x+b y)$ and expand every member by applying (2.5), (2.6) and the power series of $z /\left(e^{z}-1\right)$. We obtain

$$
\begin{aligned}
& c y \sum_{m, n=1}^{\infty} \frac{(a x+b y)^{m}(-c x)^{n} \mathfrak{S}_{m, n}}{m ! n !}\left(\begin{array}{cc}
c & a \\
b
\end{array}\right)-(a x+b y) \sum_{m, n=1}^{\infty} \frac{(c x)^{m}(c y)^{n}}{m ! n !} \mathfrak{\mathfrak { S }}_{m, n}\left(\begin{array}{cc}
a & b \\
c
\end{array}\right) \\
& +c x \sum_{m n=1}^{\infty} \frac{(-c y)^{m}(a x+b y)^{n}{ }_{\mathfrak{S}_{m, n}}}{m ! n !}\left(\begin{array}{cc}
b & c \\
a
\end{array}\right)=c^{2} x y\left[1+\sum_{\nu=1}^{\infty} B_{\nu !}(a x+b y)^{\nu}\right] \\
& -c y \sum_{\nu=1}^{\infty} \frac{B_{\nu}}{\nu ! b^{\nu-1}}\left[(a x+b y)^{\nu}+(-c x)^{\nu}\right]+c(a x+b y) \sum_{\nu=1}^{\infty} \frac{B_{\nu}}{\nu !}\left(x^{\nu}+y^{\nu}\right) \\
& -c x \sum_{\nu=1}^{\infty} \frac{B_{\nu}}{\nu ! a^{\nu-1}}\left[(-c y)^{\nu}+(a x+b y)^{\nu}\right] \text {, }
\end{aligned}
$$

this holding identically for $|x|,|y|<2 \pi$. If one uses still the binomial theorem and arranges our absolutely convergent series in terms of $x^{2}$, $y^{\nu}(\nu=1,2, \cdots)$, then comparison of the corresponding coefficients leads without difficulty to the following system of relations:

$$
\begin{array}{r}
a^{\nu} \cdot(\nu+1) b^{\nu} c \mathfrak{S}_{1, \nu}\left(\begin{array}{cc}
b & c \\
a
\end{array}\right)+b^{\nu} \sum_{\mu=1}^{\nu}(-1)^{\mu+1}\left(\begin{array}{c}
\nu+1 \\
\mu
\end{array}\right) c^{\mu} a^{\nu+1-\mu} \mathfrak{S}_{\nu+1-\mu, \mu}\left(\begin{array}{cc}
c & a \\
b
\end{array}\right) \\
+c^{\nu} \cdot(\nu+1) a b^{\nu} \mathfrak{S}_{\nu, 1}\left(\begin{array}{cc}
a & b \\
c
\end{array}\right)=B_{\nu+1}\left(a^{\nu+1}+\nu b^{\nu+1}+(-c)^{\nu+1}\right)-(\nu+1) B_{\nu}(a b)^{\nu} c \\
\nu=1,2, \cdots,
\end{array}
$$

furthermore, by $\left(\begin{array}{c}\alpha \\ \beta\end{array}\right)\left(\begin{array}{c}\gamma \\ \alpha\end{array}\right)=\left(\begin{array}{l}\gamma \\ \beta\end{array}\right)\left(\begin{array}{l}\gamma-\beta \\ \gamma-\alpha\end{array}\right)$,

$$
\begin{gathered}
a^{\nu} \cdot\left(\begin{array}{c}
\nu+1 \\
p+1
\end{array}\right) \sum_{\mu=1}^{p}(-1)^{\mu+1}\left(\begin{array}{c}
p+1 \\
\mu
\end{array}\right) b^{\nu+1-\mu} c^{\mu} \mathfrak{S}_{\mu, \nu+1-\mu}\left(\begin{array}{cc}
b & c \\
a
\end{array}\right) \\
+b^{\nu} \cdot\left(\begin{array}{c}
\nu+1 \\
p
\end{array}\right)^{\nu+1-p}(-1)^{\mu+1}\left(\begin{array}{c}
\nu+1-p \\
\mu
\end{array}\right) c^{\mu} a^{\nu+1-\mu} \mathfrak{G}_{\nu+1-\mu, \mu}\left(\begin{array}{cc}
c & a \\
b
\end{array}\right)
\end{gathered}
$$




$$
\begin{gathered}
+c^{\nu} \cdot\left[\left(\begin{array}{c}
\nu+1 \\
p+1
\end{array}\right) a^{p+1} b^{\nu-p} \mathfrak{G}_{\nu-p, p+1}\left(\begin{array}{c}
a \\
c
\end{array}\right)+\left(\begin{array}{c}
\nu+1 \\
p
\end{array}\right) a^{\nu} b^{\nu+1-p} \mathfrak{G}_{\nu+1-p, p}\left(\begin{array}{cc}
a & b \\
c
\end{array}\right)\right. \\
=B_{\nu+1}\left[\left(\begin{array}{c}
\nu+1 \\
p
\end{array}\right) a^{\nu+1}+\left(\begin{array}{c}
\nu+1 \\
p+1
\end{array}\right) b^{\nu+1}\right]-(\nu+1) B_{\nu}\left(\begin{array}{c}
\nu \\
p
\end{array}\right)(a b)^{\nu} c \\
1 \leqq p \leqq \nu-1 .
\end{gathered}
$$

The results can be written briefly in symbolic form as follows

$$
\begin{aligned}
& (\nu+1)\left[c a^{\nu \mathfrak{S}_{1, \nu}}\left(\begin{array}{cc}
b & c \\
a
\end{array}\right)+c^{\nu} a_{\mathfrak{S}} \mathfrak{\mathfrak { V }}_{\nu, 1}\left(\begin{array}{cc}
a & b \\
c
\end{array}\right)\right]-\left(a \mathfrak{S}-c_{\mathfrak{\mathfrak { S }}}\right)^{\nu+1}\left(\begin{array}{cc}
c & a \\
b
\end{array}\right) \\
& =\nu B_{\nu+1} b-(\nu+1) B_{\nu} a^{\nu} c \quad \nu=1,2, \cdots \text {, }
\end{aligned}
$$

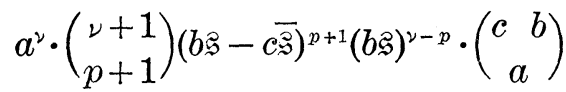

$$
\begin{aligned}
& +b^{\nu} \cdot\left(\begin{array}{c}
\nu+1 \\
p
\end{array}\right)(a \mathfrak{g}-c \overline{\mathfrak{S}})^{\nu+1-p}(a \mathfrak{s})^{p}\left(\begin{array}{cc}
c & a \\
b
\end{array}\right) \\
& -c^{\nu} \cdot\left[\left(\begin{array}{c}
\nu+1 \\
p+1
\end{array}\right) a \mathfrak{s}+\left(\begin{array}{c}
\nu+1 \\
p
\end{array}\right) b \overline{\mathfrak{g}}\right](a \mathfrak{s})^{p}(b \overline{\mathfrak{s}})^{\nu+p}\left(\begin{array}{cc}
a & b \\
c
\end{array}\right) \\
& =(p+1)\left(\begin{array}{c}
\nu+1 \\
p+1
\end{array}\right) B_{\nu} a^{\nu} b^{\nu} c \quad p=1,2, \cdots ; \nu=p+1, p+2, \cdots \text {, }
\end{aligned}
$$

where for example

$$
(b \mathfrak{G}-c \overline{\mathfrak{g}})^{p+1}(b \mathfrak{g})^{\nu-p}\left(\begin{array}{cc}
c & b \\
a
\end{array}\right)
$$

means that, after formal application of the binomial theorem to the first factor and formal multiplication by $b^{\nu-n} \cdot \mathfrak{S}^{\nu-p} \cdot\left(\begin{array}{cc}c & b \\ a\end{array}\right)$, every product ${\mathfrak{\mathfrak { S } ^ { m }}}_{\overline{\mathfrak{G}^{n}}}\left(\begin{array}{cc}c & b \\ a\end{array}\right)$ is replaced by $\mathfrak{S}_{m, n}\left(\begin{array}{cc}c & b \\ a\end{array}\right)$.

(3) We remark at once that $(5.4),(5.6)$ go over for $\nu=1$ to the reciprocity relation (5.3) and for $\nu>1$ odd, $b=1$ to the formula (cf. (1.3), (2.7))

$$
(\nu+1)\left[c a^{\nu} \cdot s_{\nu}^{(\nu)}(c, a)+c^{\nu} a, s_{\nu}^{(\nu)}(a, c)\right]=(B c-B a)^{\nu+1}+\nu B_{\nu+1}
$$

with 2

$$
(B c-B a)^{\nu+1}=\sum_{\mu=0}^{\nu+1}(-1)^{\mu}\left(\begin{array}{c}
\nu+1 \\
\mu
\end{array}\right) c^{\mu} a^{\nu+1-\mu} B_{\mu} B_{\nu+1-\mu}
$$

2 The factor $(-1)^{\mu}$ may plainly be suppressed in the last summand, that is,

$$
(B c-B a)^{\nu+1}=(B c+B a)^{\nu+1} \text {. }
$$


therefore (5.4), (5.6) generalize (5.3) and Apostol's reciprocity theorem [1, Theorem 1].

On the other hand, putting $\nu=3,5,7, \ldots$ in (5.7), we get for $c=1$

$$
\begin{aligned}
& \left(\begin{array}{c}
\nu+1 \\
p+1
\end{array}\right) a^{\nu-p}\left(s^{(\nu)}-b\right)^{p+1}(b, a)-\left(\begin{array}{c}
\nu+1 \\
p
\end{array}\right) b^{p}\left(s^{(\nu)}-a\right)^{\nu+1-p}(a, b) \\
= & \left(\begin{array}{c}
\nu+1 \\
p+1
\end{array}\right) a B_{\nu-p} B_{p+1}-\left(\begin{array}{c}
\nu+1 \\
p
\end{array}\right) b B_{\nu+1-p} B_{p},
\end{aligned}
$$

while the case $b=1$ yields

$$
\begin{aligned}
& c^{2}\left[\left(\begin{array}{c}
\nu+1 \\
p+1
\end{array}\right) a s_{\nu-p}^{(\nu)}(a, c)+\left(\begin{array}{c}
\nu+1 \\
p
\end{array}\right) s_{\nu+1-p}^{(\nu)}(a, c)\right] \\
= & \left(\begin{array}{c}
\nu+1 \\
p+1
\end{array}\right)\left(s^{(\nu)}-c\right)^{p+1}\left(a s^{(\nu)}\right)^{\nu-p}(c, a)+\left(\begin{array}{c}
\nu+1 \\
p
\end{array}\right)(a B-c \bar{B})^{\nu+1-p} B^{p},
\end{aligned}
$$

the symbolic notations being understood in similar sense as above. (5.9) and (5.10) express the first and second reciprocity law of Carlitz respectively [3, Theorems 1,2$]^{3}$, so that we have in (5.5), (5.7) a common extension of them.

6. The sum $\mathfrak{D}_{c}^{a, b}(w, z)$. We now use the generalized zeta function, defined by

$$
\zeta(z, u)=\sum_{n=0}^{\infty}(u+n)^{-z}
$$

for $\Re(z)>1$ and by analytic continuation for other values $\neq 1$ of $z, u$ denoting a fixed number with $0<u \leqq 1$. There holds the well-known formula of Hurwitz :

$$
\begin{aligned}
\zeta(z, u)=2(2 \pi)^{z-1} \Gamma(1-z) \\
\times\left(\sin \frac{\pi z}{2} \sum_{n=1}^{\infty} n^{z-1} \cos 2 n \pi u+\cos \frac{\pi z}{2} \sum_{n=1}^{\infty} n^{z-1} \sin 2 n \pi u\right) \quad \Re(z)<0 .
\end{aligned}
$$

Next we establish a functional equation for the sum

$$
\mathfrak{D}_{c}^{a, b}(w, z)=\sum_{\lambda=1}^{c-1} \zeta\left(w,\left\{\frac{\lambda a}{c}\right\}\right) \zeta\left(z,\left\{\frac{\lambda b}{c}\right\}\right)
$$

with $(a, c)=(b, c)=1, c>1$, in observing that [cf. (1.4)]

$$
\mathfrak{D}_{c}^{a, b}(1-m, 1-n)=\frac{1}{m n}\left[\mathfrak{z}_{m, n}\left(\begin{array}{cc}
a & b \\
c
\end{array}\right)-B_{m} B_{n}\right] \quad m, n=1,2, \cdots
$$

${ }^{3}$ In formula (3.2) of [3], the lack of the corresponding binomial coefficients before the Bernoullian numbers appears to be a typographical error. 
and, by $\zeta\left(z, \frac{1}{2}\right)=\left(2^{z}-1\right) \zeta(z)$ where $\zeta(z)=\zeta(z, 1)$ is Riemann's zeta function,

$$
\mathfrak{D}_{2}^{a, b}(w, z)=\left(2^{w}-1\right)\left(2^{z}-1\right) \cdot \zeta(w) \zeta(z) .
$$

Theorem 2. For $(a, c)=(b, c)=1, c>2$ and for any $w, z$ distinct from 0 and 1 we have the relation

$$
\begin{gathered}
\mathfrak{D}_{c}^{a, b}(w, z)=\left(c^{w+z}-1\right) \zeta(w)_{\circ}^{\prime}(z)+\pi^{-1}(2 c \pi)^{w+z-1} \Gamma(1-w) \Gamma(1-z) \\
\times\left\{\cos \frac{\pi}{2}(w-z) D_{c}^{b, a}(1-w, 1-z)-\cos \frac{\pi}{2}(w+z) D_{c}^{b,-a}(1-w, 1-z)\right\} .
\end{gathered}
$$

Proof. $1^{\circ} \quad$ First let $\Re(w)<0, \Re(z)<0$. We transform

$$
\overline{\mathfrak{D}}_{c}^{a, b}(w, z)=\sum_{\lambda=1}^{c} \zeta\left(w,\left\{\frac{\lambda a}{c}\right\}\right) \zeta\left(z,\left\{\frac{\lambda b}{c}\right\}\right)
$$

by means of (6.1).

Since the series involved in Hurwitz's formula are absolutely convergent, one obtains after substitution into (6.6)

$$
\begin{aligned}
& \bar{D}_{c}^{a, b}(w, z)=4(2 \pi)^{w+z-2} \Gamma(1-w) \Gamma(1-z) \\
\times & \sum_{m, n=1}^{\infty} m^{w-1} n^{z-1}\left(\phi_{m, n} \cdot \sin \frac{\pi w}{2} \sin \frac{\pi z}{2}+\psi_{m, n} \cdot \cos \frac{\pi w}{2} \cos \frac{\pi z}{2}\right),
\end{aligned}
$$

where

$$
\begin{aligned}
& \phi_{m, n}=\sum_{\mu=1}^{c} \cos 2 m \pi \frac{\mu a}{c} \cos 2 n \pi \frac{\mu b}{c}=\left\{\begin{array}{l}
c, \text { if } c \mid a m \pm b n, \\
0 \text { for } c \nmid a m \pm b n, \\
c / 2 \text { otherwise },
\end{array}\right. \\
& \phi_{m, n}=\sum_{\mu=1}^{c} \sin 2 m \pi \frac{\mu a}{c} \sin 2 n \pi \frac{\mu b}{c}=\left\{\begin{array}{r}
c / 2, \text { if } c \mid a m-b n \text { but } \\
c \nmid a m+b n, \\
-c / 2, \text { if } c \mid a m+b n \text { and } \\
c \nmid a m-b n, \\
0 \text { otherwise } .
\end{array}\right.
\end{aligned}
$$

Hence it follows easily that

$$
\begin{aligned}
& \overline{\mathfrak{D}}_{c}^{a, b}(w, z)=2 c(2 \pi)^{w+z-2} \Gamma(1-w) \Gamma(1-z) \cdot\left\{2 \sin \frac{\pi w}{2} \sin \frac{\pi z}{2} \sum_{c|m, c| n} m^{w-1} n^{z-1}\right. \\
& \left.+\cos \frac{\pi}{2}(w-z) \sum_{\substack{a m \equiv m(\bmod c) \\
c \nmid m, c \nmid n}} m^{w-1} n^{z-1}-\cos \frac{\pi}{2}(w+z) \sum_{\substack{a m=-b n(\bmod c) \\
c \nmid m, b \nmid n}} m^{w-1} n^{z-1}\right\} .
\end{aligned}
$$

Now, by the functional equation of $\zeta(s)$ we have 


$$
\begin{aligned}
4 c(2 \pi)^{w+z-2} \Gamma(1-w) \Gamma(1-z) \sin \frac{\pi w}{2} \sin \frac{\pi z}{2} \sum_{c|m, c| n} m^{w-1} n^{z-1} \\
=c^{w+z-1} \zeta(w) \zeta(z) .
\end{aligned}
$$

Furthermore, ar $(r=0,1, \cdots, c-1)$ and $b r(r=0,1, \cdots, c-1)$ being complete systems of residues $\bmod c$, we can write

$$
\begin{aligned}
& \sum_{\substack{a m \equiv b n(\bmod c) \\
c \nmid m, c \nmid n}} m^{w-1} n^{z-1}=\sum_{r=1}^{c-1}\left(\sum_{m \equiv r b(\bmod c)} m^{w-1}\right)\left(\sum_{n \equiv r a(\bmod c)} n^{z-1}\right) \\
& =c^{w+z-2} \sum_{r=1}^{c-1}\left[\sum_{M=0}^{\infty}\left(\left\{\begin{array}{c}
r b \\
c
\end{array}\right\}+M\right)^{w-1}\right]\left[\sum_{N=1}^{\infty}\left(\left\{\frac{r a}{c}\right\}+N\right)^{z-1}\right] \\
& =c^{w+z-2} \sum_{r=1}^{c-1} \zeta\left(1-w,\left\{\frac{r b}{c}\right\}\right) \zeta\left(1-z,\left\{\frac{r a}{c}\right\}\right)
\end{aligned}
$$

and similarly

$$
\begin{aligned}
\sum_{\substack{a m \equiv=-b n(\bmod c) \\
c \nmid m, c \nmid m}} m^{w-1} n^{z-1} & =\sum_{r=1}^{c-1}\left(\sum_{m \equiv r b(\bmod c)} m^{w-1}\right)\left(\sum_{n \equiv-r a(\bmod c)} n^{z-1}\right) \\
& =c^{w+z-2} \sum_{r=1}^{c-1} \zeta\left(1-w,\left\{\frac{r b}{c}\right\}\right) \zeta\left(1-z,\left\{\frac{r a}{c}\right\}\right) .
\end{aligned}
$$

$(6.10)-(6.13)$ yield together

$$
\begin{gathered}
\overline{\mathfrak{D}}_{c}^{a, b}(w, z)=c^{w+z-1} \zeta(w) \zeta(z)+\pi^{-1}(2 c \pi)^{w+z-1} \Gamma(1-w) \Gamma(1-z) \\
\times\left\{\cos \frac{\pi}{2}(w-z) \mathfrak{D}_{c}^{b, a}(1-w, 1-z)-\cos \frac{\pi}{2}(w+z) \mathfrak{D}_{c}^{b,-a}(1-w, 1-z)\right\} .
\end{gathered}
$$

$2^{\circ}$ Finally, (6.5) follows immediately from (6.14), in view of

$$
\mathfrak{D}_{c}^{a, b}(w, z)=\overline{\mathfrak{D}}_{c}^{a, b}(w, z)-\zeta(w) \zeta(z) \quad \mathfrak{R}(w)<0, \mathfrak{R}(z)<0
$$

and by analytic continuation.

7. Some remarks. In [2], Apostol finds certain finite sum representations for $s_{\nu}^{(\nu)}(h, k)$, involving cotangents, $\zeta(z, u), \Gamma^{\prime}(z) / \Gamma(z)$ and he uses these expressions to give a short analytic proof of (5.8) [Theorems $1,2]$. It may be noted that the above Theorem 2 implies the results in question, arising as limiting cases for $w \rightarrow 0$, and $z \rightarrow 0, z=-1$, $-2, \cdots$.

The form of $\mathfrak{S}_{c}^{a, b}(x, y), \mathfrak{D}_{c}^{a, b}(w, z)$ suggests applications in connection with certain Lambert series, generalizing those investigated by Rademacher, Apostol and Carlitz. I hope to return on this problem in another paper. 


\section{REFERENCES}

1. T. M. Apostol, Generalized Dedekind sums and transformation formulae of certain Lambert series, Duke Math. J., 17 (1950), 147-157.

2. __ Theorems on generalized Dedekind sums, Pacific J. Math., 2 (1952), 1-9.

3. L. Carlitz, Some theorems on generalized Dedekind sums, Pacific J. Math., 3 (1953), 513-522.

4. __ The reciprocity theorem for Dedekind sums, Pacific J. Math., 3 (1953), 523527.

5. __ A note on generalized Dedekind sums, Duke Math. J., 21 (1954), 399-403.

$6 . \quad \ldots \ldots$ - Dedekind sums and Lambert series, Proc. Amer. Math. Soc., 5 (1954), 580584.

7. R. Dedekind, Erläuterungen zu Riemann's Fragmenten über die Grenzfälle der elliptischen Funktionen, Gesammelte mathematische Werke, vol. 1, Braunschweig, 1930, 159173.

8. L. J. Mordell, The reciprocity formula for Dedekind sums, Amer. J. Math. 73 (1951), 593-598.

9. H. Rademacher, Zur Theorie der Modulfunktionen, J. Reine Angew. Math., 167 (1932), 312-336.

10.

Die Reziprozitätsformel für Dedekindsche Summen, Acta Sci. Math. Szeged, 12B (1950), 57-60.

11. Generalization of the reciprocity formula for Dedekind sums, Duke Math. J., 21 (1954), 391-397.

12. H. Rademacher and A. Whiteman, Theorems on Dedekind sums, Amer. J. Math., 63 (1941), 377-407.

13. L. Rédei, Elementarer Beweis und Verallgemeinerung einer Reziprozitätsformel von Dedekind, Acta Sci. Math. Szeged, 12B (1950), 236-239.

EÖTVÖs LORÁND UNIVERSITY, BUdAPEST, HuNGARY. 


\section{PACIFIC JOURNAL OF MATHEMATICS}

\section{EDITORS}

H. L. ROYDEN

Stanford University

Stanford, California

R. A. Beaumont

University of Washington

Seattle 5, Washington

\section{A. L. Whiteman}

University of Southern California

Los Angeles 7, California

E. G. Straus

University of California

Los Angeles 24, California

\section{ASSOCIATE EDITORS}
E. F. BECKENBACH
C. E. BURGESS
M. HALL
E. HEWITT

\author{
A. HORN \\ V. GANAPATHY IYER \\ R. D. JAMES \\ M. S. KNEBELMAN
}

L. NACHBIN

I. NIVEN

G. SZEKERES

T. G. OSTROM

M. M. SCHIFFER
F. WOLF

K. YOSIDA

\section{SUPPORTING INSTITUTIONS}

\author{
UNIVERSITY OF BRITISH COLUMBIA \\ CALIFORNIA INSTITUTE OF TECHNOLOGY \\ UNIVERSITY OF CALIFORNIA \\ MONTANA STATE UNIVERSITY \\ UNIVERSITY OF NEVADA \\ OREGON STATE COLLEGE \\ UNIVERSITY OF OREGON \\ UNIVERSITY OF SOUTHERN CALIFORNIA
}

\author{
STANFORD UNIVERSITY \\ UNIVERSITY OF UTAH \\ WASHINGTON STATE COLLEGE \\ UNIVERSITY OF WASHINGTON \\ AMERICAN MATHEMATICAL SOCIETY \\ CALIFORNIA RESEARCH CORPORATION \\ HUGHES AIRCRAFT COMPANY \\ THE RAMO-WOOLDRIDGE CORPORATION
}

Mathematical papers intended for publication in the Pacific Journal of Mathematics should be typewritten (double spaced), and the author should keep a complete copy. Manuscripts may be sent to any of the editors. All other communications to the editors should be addressed to the managing editor, E. G. Straus at the University of California, Los Angeles 24, California.

50 reprints per author of each article are furnished free of charge; additional copies may be obtained at cost in multiples of 50 .

The Pacific Journal, of Mathematics is published quarterly, in March, June, September, and December. The price per volume (4 numbers) is $\$ 12.00$; single issues, $\$ 3.50$. Back numbers are available. Special price to individual faculty members of supporting institutions and to individual members of the American Mathematical Society: $\$ 4.00$ per volume; single issues, $\$ 1.25$.

Subscriptions, orders for back numbers, and changes of address should be sent to Pacific Journal of Mathematics, 2120 Oxford Street, Berkeley 4, California.

Printed at Kokusai Bunken Insatsusha (International Academic Printing Co., Ltd.), No. 10, 1-chome, Fujimi-cho, Chiyoda-ku, Tokyo, Japan.

PUBLISHED BY PACIFIC JOURNAL OF MATHEMATICS, A NON-PROFIT CORPORATION

The Supporting Institutions listed above contribute to the cost of publication of this Journal, but they are not owners or publishers and have no responsibility for its content or policies. 


\section{Pacific Journal of Mathematics}

\section{Vol. 7, No. 2 \\ February, 1957}

William F. Donoghue, Jr., The lattice of invariant subspaces of a completely continuous quasi-nilpotent transformation ................... 1031

Michael (Mihály) Fekete and J. L. Walsh, Asymptotic behavior of restricted extremal polynomials and of their zeros.................... 1037

Shaul Foguel, Biorthogonal systems in Banach spaces ............... 1065

David Gale, A theorem on flows in networks ................... 1073

Ioan M. James, On spaces with a multiplication .................. 1083

Richard Vincent Kadison and Isadore Manual Singer, Three test problems in operator theory .................................... 1101

Maurice Kennedy, A convergence theorem for a certain class of Markoff processes........................................ 1107

G. Kurepa, On a new reciprocity, distribution and duality law ........ 1125

Richard Kenneth Lashof, Lie algebras of locally compact groups ........ 1145

Calvin T. Long, Note on normal numbers .................... 1163

M. Mikolás, On certain sums generating the Dedekind sums and their reciprocity laws ..................................... 1167

Barrett O'Neill, Induced homology homomorphisms for set-valued maps......................................... 1179

Mary Ellen Rudin, A topological characterization of sets of real numbers........................................... 1185

M. Schiffer, The Fredholm eigen values of plane domains 1187

F. A. Valentine, A three point convexity property .........

Alexander Doniphan Wallace, The center of a compact lattice is totally



Alexander Doniphan Wallace, Two theorems on topological lattices.

G. T. Whyburn, Dimension and non-density preservation of mappings...

John Hunter Williamson, On the functional representation of certain algebraic systems ... 